\title{
Fractional Variational Iteration Method for Fractional Cauchy Problems
}

\author{
Bao Si-yuan \\ Department of Engineering Mechanics, College of Civil Engineering, Suzhou University of Science and Technology, \\ Suzhou 215011, China \\ Correspondence should be addressed to Bao Si-yuan; bsiyuan@126.com
}

Received 24 August 2013; Accepted 14 January 2014; Published 23 February 2014

Academic Editor: Ashraf M. Zenkour

Copyright (C) 2014 Bao Si-yuan. This is an open access article distributed under the Creative Commons Attribution License, which permits unrestricted use, distribution, and reproduction in any medium, provided the original work is properly cited.

The fractional variational iteration method is used to solve the fractional Cauchy problem. Some examples are given to elucidate the solution procedure and reliability of the obtained results. The variational iteration algorithm leads to exact solutions in the present study.

\section{Introduction}

Fractional problems have attracted many scholars' attention as the actual problems can be much better simulated by using the fractional derivatives than by using traditional integral derivatives [1-6].

In [7], the author gave a very lucid as well as elementary discussion of the variational iteration method. The variational iteration algorithm [7-14] is very simple, with results of high precision (sometimes exact solutions can be obtained), and is easy to understand and has been widely applied in various nonlinear problems. In addition, many authors have made a great effort to give sophisticated theoretical verification of the variational iteration method; for example, Odibat [15], Salkuyeh [16], and Tatari and Dehghan [17] proved that the variational iteration algorithm leads to convergent results. Due to its flexibility and ability to solve nonlinear equations accurately and conveniently, the method has been modified or improved to solve nonlinear problems more efficiently [1821]; furthermore the method has been extended to handle fractional nonlinear models and the fractional variational iteration method (FVIM) has been presented [22].

Many scholars have applied FVIM to solve linear or nonlinear fractional order differential equations [23-28]. For a relatively comprehensive survey on the concepts, theory, and applications of the method, readers are referred to review articles [10, 29].
In [22] the improved VIM is called fractional variational iteration method and the new Lagrange multiplier is determined by using the Laplace transformation. In this paper, we use the fractional variational iteration method to discuss the fractional-order partial differential equation in the form

$$
\begin{gathered}
u_{t}^{(\alpha)}(x, t)+a(x, t) u_{x}(x, t)=\phi(x), \quad x \in R, t>0, \\
u(x, 0)=\psi(x), \quad x \in R .
\end{gathered}
$$

When $a(x, t)=a$ is a constant and $\phi(x)=0,(1)$ is a linear equation called the fractional transport equation which can describe many interesting phenomena such as the spread of AIDS and the moving of wind. When $a(x, t)=u(x, t)$, the equation is called the nefractional inviscid Burgers' equation arising in a one-dimensional stream of particles or fluid having zero viscosity. When $\alpha=1,(1)$ is the equation for traditional Cauchy problem [30, 31].

\section{The Fractional Variational Iteration Method}

To discuss the fractional problems, two definitions are introduced. 
Definition 1. The Caputo derivative is given as

$$
\begin{array}{r}
{ }_{0}^{C} D_{t}^{\alpha} f(t)=\frac{1}{\Gamma(m-\alpha)} \int_{a}^{t}(t-\tau)^{m-\alpha-1} f^{(m)}(\tau) \mathrm{d} \tau, \\
t>a, \quad m-1<\alpha<m \in Z^{+} .
\end{array}
$$

Definition 2. The Riemann-Liouville (R-L) integration of $f(t)$ is defined as

$$
I_{a, t}^{\alpha} f(t)=\frac{1}{\Gamma(\alpha)} \int_{a}^{t}(t-\tau)^{\alpha-1} f(\tau) \mathrm{d} \tau, \quad \alpha>0 .
$$

To illustrate the basic concepts of the fractional variational iteration method, one considers the following general nonlinear fractional system:

$$
{ }_{0}^{C} D_{t}^{\alpha} u+R[u]+N[u]=f(\tau)
$$

where $R[u]$ is a linear term and $N[u]$ is a nonlinear one and $f(t)$ is a known analytic function.

According to the fractional variational iteration method, a correction functional can be constructed as follows:

$$
\begin{aligned}
u_{n+1}= & u_{n} \\
& +\int_{0}^{t} \lambda(t, \tau)\left[{ }_{0}^{C} D_{t}^{\alpha} u_{n}+R\left[u_{n}\right]+N\left[u_{n}\right]-f(\tau)\right] \mathrm{d} \tau,
\end{aligned}
$$

where $\lambda(t, \tau)$ is a general Lagrange multiplier [7-12], which can be identified optimally via the variational theory, the subscript $n$ denotes the $n$th approximation, and $R\left[u_{n}\right]$ and $N\left[u_{n}\right]$ are considered as a restricted variation [7]; that is $\delta R\left[u_{n}\right]=0, \delta N\left[u_{n}\right]=0$.

Taking Laplace transform on the correction functional equation established via the R-L integration, Wu and Baleanu [22] present a new way to identify the Lagrange multiplier. The Lagrange multiplier can be identified as

$$
\lambda(t, \tau)=\frac{(-1)^{\alpha}(\tau-t)^{\alpha-1}}{\Gamma(\alpha)} .
$$

The variational iteration formula (6) can be improved as

$$
\begin{aligned}
u_{n+1}=u_{n}+\int_{0}^{t} \frac{(-1)^{\alpha}(\tau-t)^{\alpha-1}}{\Gamma(\alpha)} & {\left[{ }_{0}^{C} D_{t}^{\alpha} u_{n}+R\left[u_{n}\right]\right.} \\
& \left.+N\left[u_{n}\right]-f(\tau)\right] \mathrm{d} \tau
\end{aligned}
$$

The initial guess $u_{0}$ can be freely chosen with possible unknown constants; it can also be solved from its corresponding linear homogeneous equation.

The above iteration formula (8) is also valid for differential equations when $\alpha$ is an arbitrary positive integer.

The fractional variational iteration method can solve effectively, easily, and accurately a large class of nonlinear fractional problems with approximations converging rapidly to the accurate solution.

\section{Applications}

Since our focus is on the ideas and basic principles, we will consider only the simplest possible equations to clearly illustrate the solution procedure. In particular, we will focus on pure Cauchy problems. These problems are initial value problems.

According to (8), we can construct a correction functional to (1) which reads

$$
\begin{aligned}
u_{n+1}(x, t)= & u_{n}(x, t) \\
+\int_{0}^{t} \frac{(-1)^{\alpha}(\tau-t)^{\alpha-1}}{\Gamma(\alpha)} & {\left[{ }_{0}^{C} D_{t}^{\alpha} u_{n}(x, \tau)\right.} \\
& +a(x, \tau) u_{x}(x, \tau) \\
& -\phi(x)] \mathrm{d} \tau .
\end{aligned}
$$

Example 3. Consider the fractional transport equation:

$$
\begin{gathered}
u_{t}^{(\alpha)}(x, t)+a u_{x}(x, t)=0, \quad x \in R, t>0, \\
u(x, 0)=x^{2}, \quad x \in R .
\end{gathered}
$$

According to the iteration formula (8), we can obtain

$$
\begin{array}{r}
u_{n+1}=u_{n}-\int_{0}^{t} \frac{(\tau-t)^{\alpha}}{\Gamma(\alpha)}\left[u_{n \tau}^{(\alpha)}(x, \tau)+a u_{n x}(x, t)\right] \mathrm{d} \tau, \\
x \in R, \quad t>0 .
\end{array}
$$

The initial iterative value is selected as $u_{0}(x, 0)=u(x, 0)=$ $x^{2}$ from the given initial condition. Using (10), we obtain the following successive approximations:

$$
\begin{aligned}
& u_{1}(x, t)=x^{2}-2 a x \frac{t^{\alpha}}{\Gamma(\alpha+1)} \\
& u_{2}(x, t)=x^{2}-2 a x \frac{t^{\alpha}}{\Gamma(\alpha+1)}+2 a^{2} \frac{t^{2 \alpha}}{\Gamma(2 \alpha+1)} \\
& u_{3}(x, t)=x^{2}-2 a x \frac{t^{\alpha}}{\Gamma(\alpha+1)}+2 a^{2} \frac{t^{2 \alpha}}{\Gamma(2 \alpha+1)}
\end{aligned}
$$

$$
u_{n}(x, t)=x^{2}-2 a x \frac{t^{\alpha}}{\Gamma(\alpha+1)}+2 a^{2} \frac{t^{2 \alpha}}{\Gamma(2 \alpha+1)} .
$$

So the exact analytical solution is yielded as

$$
u(x, t)=x^{2}-2 a x \frac{t^{\alpha}}{\Gamma(\alpha+1)}+2 a^{2} \frac{t^{2 \alpha}}{\Gamma(2 \alpha+1)} .
$$

Example 4. Consider the fractional nonlinear Cauchy problem

$$
\begin{gathered}
u_{t}^{(\alpha)}(x, t)+x u_{x}(x, t)=0, \quad x \in R, t>0 \\
u_{0}(x, 0)=u(x, 0)=x^{2}, \quad x \in R .
\end{gathered}
$$


According to the iteration formula (8), we can obtain

$$
\begin{array}{r}
u_{n+1}=u_{n}-\int_{0}^{t} \frac{(\tau-t)^{\alpha}}{\Gamma(\alpha)}\left[u_{t}^{(\alpha)}(x, t)+x u_{x}(x, t)\right] \mathrm{d} \xi \\
x \in R, \quad t>0 .
\end{array}
$$

The initial iterative value is selected as $u_{0}(x, 0)=u(x, 0)=$ $x^{2}$ from the given initial condition. Using (15), we obtain the following successive approximations:

$$
\begin{aligned}
& u_{0}(x, t)=x^{2} \\
& u_{1}(x, t)=x^{2}-2 x^{2} \frac{t^{\alpha}}{\Gamma(\alpha+1)} \\
& \begin{aligned}
u_{2}(x, t)= & x^{2}-2 x^{2} \frac{t^{\alpha}}{\Gamma(\alpha+1)}+x^{2} \frac{\left(2 t^{\alpha}\right)^{2}}{\Gamma(2 \alpha+1)} \\
u_{3}(x, t)= & x^{2}-2 x^{2} \frac{t^{\alpha}}{\Gamma(\alpha+1)}+x^{2} \frac{\left(2 t^{\alpha}\right)^{2}}{\Gamma(2 \alpha+1)}-x^{2} \frac{\left(2 t^{\alpha}\right)^{3}}{\Gamma(3 \alpha+1)} \\
\vdots & \quad\left[\begin{array}{l}
2 t^{\alpha} \\
u_{n}(x, t)=
\end{array} x^{2}+\frac{\left(2 t^{\alpha}\right)^{2}}{\Gamma(2 \alpha+1)}-\frac{\left(2 t^{\alpha}\right)^{3}}{\Gamma(3 \alpha+1)}\right. \\
& \left.\quad+\frac{\left(2 t^{\alpha}\right)^{4}}{\Gamma(4 \alpha+1)}-\frac{\left(2 t^{\alpha}\right)^{5}}{\Gamma(5 \alpha+1)}+\cdots\right] .
\end{aligned}
\end{aligned}
$$

The VIM admits the use of $u=\lim _{n \rightarrow \infty} u_{n}$, which gives the exact solution

$$
u(x, t)=x^{2} E_{\alpha}\left(-2 t^{\alpha}\right)
$$

where $E_{\alpha}$ is Mittag-Leffler function

$$
E_{\alpha}(z)=\sum_{n=0}^{\infty} \frac{z^{n}}{\Gamma(\alpha n+1)}
$$

Example 5. Consider the following nonhomogeneous fractional Cauchy problem:

$$
\begin{gathered}
u_{t}^{(\alpha)}(x, t)+x u_{x}(x, t)=x, \quad x \in R, t>0 \\
u(x, 0)=e^{x}, \quad x \in R .
\end{gathered}
$$

According to the iteration formula (9), we can obtain

$$
u_{n+1}=u_{n}-\int_{0}^{t} \frac{(\tau-t)^{\alpha}}{\Gamma(\alpha)}\left[u_{t}^{(\alpha)}(x, t)+x u_{x}(x, t)-x\right] \mathrm{d} \xi .
$$

The following successive approximations are obtained by using (20) with the selected initial value $u_{0}=e^{x}$ :

$$
\begin{aligned}
u_{1}(x, t)= & e^{x}+\left(x-e^{x}\right) \frac{t^{\alpha}}{\Gamma(\alpha+1)} \\
u_{2}(x, t)= & x^{2}+\left(x-e^{x}\right) \frac{t^{\alpha}}{\Gamma(\alpha+1)}+\left(e^{x}-1\right) \frac{t^{2 \alpha}}{\Gamma(2 \alpha+1)} \\
u_{3}(x, t)= & x^{2}+\left(x-e^{x}\right) \frac{t^{\alpha}}{\Gamma(\alpha+1)}+\left(e^{x}-1\right) \frac{t^{2 \alpha}}{\Gamma(2 \alpha+1)} \\
& -e^{x} \frac{t^{3 \alpha}}{\Gamma(3 \alpha+1)} \\
\vdots & \left.\quad+\frac{\Gamma(\alpha+1) t^{\alpha}}{\Gamma(2 \alpha+1)}\right] \frac{t^{\alpha}}{\Gamma(\alpha+1)} \\
u_{n}(x, t)= & +e^{x}\left[1-\frac{t^{\alpha}}{\Gamma(\alpha+1)}+\frac{t^{2 \alpha}}{\Gamma(2 \alpha+1)}-\frac{t^{3 \alpha}}{\Gamma(3 \alpha+1)}\right. \\
& \left.+\frac{t^{4 \alpha}}{\Gamma(4 \alpha+1)}-\frac{t^{5 \alpha}}{\Gamma(5 \alpha+1)}+\cdots\right] .
\end{aligned}
$$

By using $u=\lim _{n \rightarrow \infty} u_{n}$, the exact solution of the equation is obtained as

$$
u(x, t)=\left[x-\frac{\Gamma(\alpha+1) t^{\alpha}}{\Gamma(2 \alpha+1)}\right] \frac{t^{\alpha}}{\Gamma(\alpha+1)}+e^{x} E_{\alpha}\left(-t^{\alpha}\right) .
$$

Particularly when $\alpha=1$, the result in (22) is $u(x, t)=(x-$ $(t / 2)) t+e^{x-t}$, which is the same as that in [30].

Example 6. Consider the fractional inviscid Burgers' equation

$$
\begin{gathered}
u_{t}^{(\alpha)}(x, t)+u(x, t) u_{x}(x, t)=0, \quad x \in R, t>0, \\
u(x, 0)=x, \quad x \in R .
\end{gathered}
$$

According to the iteration formula (8), we can obtain

$$
u_{n+1}=u_{n}-\int_{0}^{t} \frac{(\tau-t)^{\alpha}}{\Gamma(\alpha)}\left[u_{t}^{(\alpha)}(x, \tau)+u(x, \tau) u_{x}(x, \tau)\right] \mathrm{d} \tau
$$


Starting with initial approximation $u_{0}(x, 0)=u(x, 0)=x$ and proceeding in a similar way illustrated above, we obtain the following successive approximations:

$$
\begin{aligned}
u_{0}(x, t)= & 1-\frac{t^{\alpha}}{\Gamma(\alpha+1)} \\
u_{1}(x, t)= & 1-\frac{t^{\alpha}}{\Gamma(\alpha+1)}+2 \frac{t^{2 \alpha}}{\Gamma(2 \alpha+1)}-\frac{\Gamma(2 \alpha+1) t^{3 \alpha}}{\Gamma(3 \alpha+1) \Gamma^{2}(\alpha+1)} \\
u_{2}(x, t)= & 1-\frac{t^{\alpha}}{\Gamma(\alpha+1)}+2 \frac{t^{2 \alpha}}{\Gamma(2 \alpha+1)}+a_{3} \frac{t^{3 \alpha}}{\Gamma(3 \alpha+1)} \\
& +a_{4} \frac{t^{4 \alpha}}{\Gamma(4 \alpha+1)}+a_{5} \frac{t^{5 \alpha}}{\Gamma(5 \alpha+1)}+a_{6} \frac{t^{6 \alpha}}{\Gamma(6 \alpha+1)} \\
& +a_{7} \frac{t^{7 \alpha}}{\Gamma(7 \alpha+1)} \\
\vdots &
\end{aligned}
$$

where

$$
\begin{aligned}
& a_{3}=-\frac{4}{\Gamma(1+3 \alpha)}-\frac{\Gamma(1+2 \alpha)}{\Gamma^{2}(1+\alpha) \Gamma(1+3 \alpha)} \\
& a_{4}=\frac{2}{\alpha \Gamma(4 \alpha)}+\frac{\Gamma(2 \alpha)}{\alpha^{2} \Gamma^{2}(\alpha) \Gamma(4 \alpha)}+\frac{3 \Gamma(3 \alpha)}{2 \alpha^{2} \Gamma(\alpha) \Gamma(2 \alpha) \Gamma(4 \alpha)} \\
& a_{5}=-\frac{4 \Gamma(1+4 \alpha)}{\Gamma^{2}(1+2 \alpha)}-\frac{2 \Gamma(1+2 \alpha) \Gamma(1+4 \alpha)}{\Gamma^{3}(1+\alpha) \Gamma(1+3 \alpha)} \\
& a_{6}=\frac{4 \Gamma(1+5 \alpha)}{\Gamma^{2}(1+\alpha) \Gamma(1+3 \alpha)} \\
& a_{7}=-\frac{\Gamma^{2}(1+2 \alpha) \Gamma(1+6 \alpha)}{\Gamma^{4}(1+\alpha) \Gamma^{2}(1+3 \alpha)} .
\end{aligned}
$$

Particularly for the case $\alpha=1$, the result for $u_{3}$ in (23) will be

$$
u_{3}=x\left(1-t+t^{2}-t^{3}+\frac{2}{3} t^{4}-\frac{2}{3} t^{5}+\frac{1}{9} t^{6}-\frac{1}{63} t^{7}\right)
$$

which is the same as that in [30].

In order to show the convergence of the iteration solutions, we introduce a function $v_{i}(x, t)$ :

$$
v_{i}(x, t)=\frac{u_{i}(x, t)}{x} .
$$

It can be seen that $v_{i}$ is a function of $t$. When $\alpha=1$, the accurate solution of $(23)$ is $u=x /(1+t)$; then $v=1 /(1+t)$.

Figures 1,2, and 3 show the curves of $v_{i}$ (where $i$ means the iteration times) changing with time $t$ when $\alpha=0.5,1$, and 1.5. When $\alpha=1$, the fifth iteration result $v_{5}$ is almost the same as the exact solution at $t \in[0,1]$. When $\alpha=1.5$, the iteration results converge fast and the fourth iteration result $v_{4}$ is almost the same as $v_{5}$ at $t \in[0,3]$.

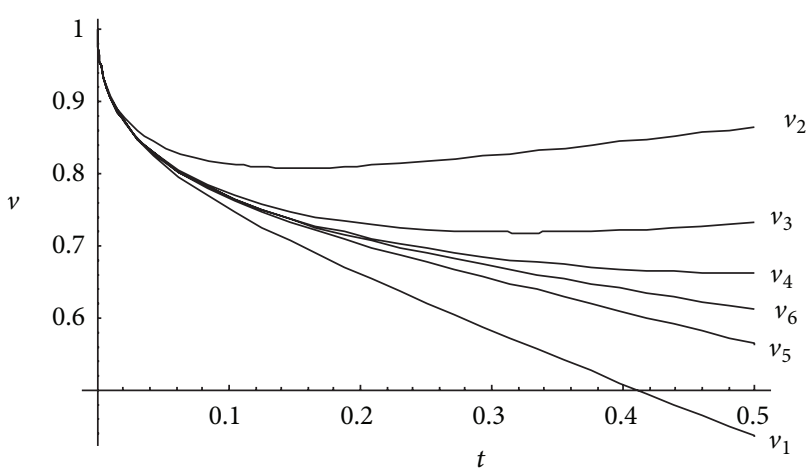

Figure 1: $v_{i}(t)$ 's curve changing with time $t$ when $\alpha=0.5$.

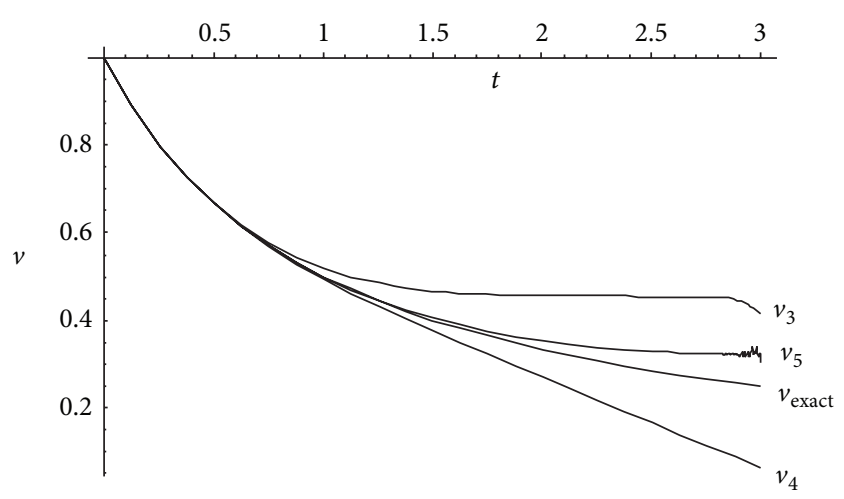

FIGURE 2: $v_{i}(t)$ 's curve changing with time $t$ when $\alpha=1$.

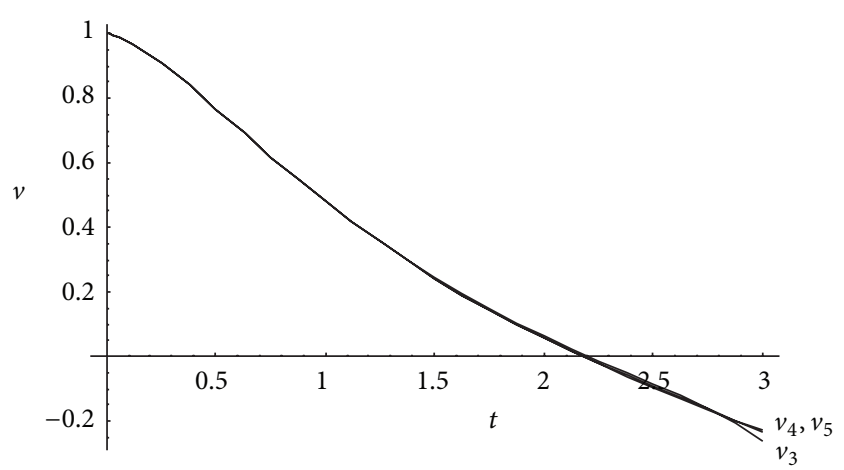

FIGURE 3: $v_{i}(t)$ 's curve changing with time $t$ when $\alpha=1.5$.

\section{Conclusions}

The variational iteration method has been proved by many authors to be a powerful mathematical tool for various kinds of nonlinear problems.

In this paper, we extend the use of fractional variational iteration method to fractional Cauchy problems and give the numerical examples. Compared with the classical VIM, the modified version method is powerful for solving differential equations with fractional derivatives. The higher order approximate solutions of the Cauchy equation illustrate the method's efficiency and high accuracy. 


\section{Conflict of Interests}

The author declares that there is no conflict of interests regarding the publication of this paper.

\section{Acknowledgments}

The author would like to thank the reviewers for their careful reading and helpful comments. The work is supported by the National Natural Science Foundation of China (Grant no. 11202146).

\section{References}

[1] W. Chen, H. G. Sun, and X. C. Li, Modeling Using the Fractional Derivative in Mechanics and Engineering Problems, Science Press, Beijing, China, 1st edition, 2010, (Chinese).

[2] K. S. Miller and B. Ross, An Introduction to the Fractional Calculus and Fractional Differential Equations, Wiley, New York, NY, USA, 1993.

[3] K. B. Oldham and J. Spanier, The Fractional Calculus Theory and Applications of Differentiation and Integration to Arbitrary Order, Academic Press, New York, NY, USA, 1974.

[4] L. Debnath, "Fractional integrals and fractional differential equations in fluid mechanics," Fractional Calculus \& Applied Analysis, vol. 6, pp. 119-155, 2003.

[5] I. Podlubny, Fractional Differential Equations, Academic Press, New York, NY, USA, 1999.

[6] A. A. Kilbas, H. M. Srivastava, and J. J. Trujillo, Theory and Applications of Fractional Differential Equations, Elsevier, Amsterdam, The Netherlands, 2006.

[7] J. H. He, "Approximate analytical solution for seepage flow with fractional derivatives in porous media," Computer Methods in Applied Mechanics and Engineering, vol. 167, no. 1-2, pp. 57-68, 1998.

[8] J. H. He, "Variational iteration method-a kind of non-linear analytical technique: some examples," International Journal of Non-Linear Mechanics, vol. 34, no. 4, pp. 699-708, 1999.

[9] J. H. He, "Variational iteration method-some recent results and new interpretations," Journal of Computational and Applied Mathematics, vol. 207, pp. 3-17, 2007.

[10] J. H. He and X. H. Wu, "Variational iteration method: new development and applications," Computers \& Mathematics with Applications, vol. 54, no. 7-8, pp. 881-894, 2007.

[11] J. H. He, G.-C. Wu, and F. Austin, "The variational iteration method which should be followed," Nonlinear Science Letters A, vol. 1, pp. 1-30, 2010.

[12] M. Jia-Qi, Z. Wei-Jiang, and C. Xian-Feng, "Variational iteration method for solving a class of strongly nonlinear evolution equations," Acta Physica Sinica, vol. 58, no. 11, pp. 7397-7401, 2009 (Chinese).

[13] M. A. Noor and S. T. Mohyud-Din, "Variational iteration method for solving higher-order nonlinear boundary value problems using He's polynomials," International Journal of Nonlinear Sciences and Numerical Simulation, vol. 9, no. 2, pp. 141-156, 2008.

[14] A. M. Wazwaz, "A study on linear and nonlinear Schrodinger equations by the variational iteration method," Chaos, Solitons and Fractals, vol. 37, no. 4, pp. 1136-1142, 2008.
[15] Z. M. Odibat, "A study on the convergence of variational iteration method," Mathematical and Computer Modelling, vol. 51, no. 9-10, pp. 1181-1192, 2010.

[16] D. K. Salkuyeh, "Convergence of the variational iteration method for solving linear systems of ODEs with constant coefficients," Computers \& Mathematics with Applications, vol. 56, no. 8, pp. 2027-2033, 2008.

[17] M. Tatari and M. Dehghan, "On the convergence of He's variational iteration method," Journal of Computational and Applied Mathematics, vol. 207, no. 1, pp. 121-128, 2007.

[18] F. Geng, "A modified variational iteration method for solving Riccati differential equations," Computers \& Mathematics with Applications, vol. 60, no. 7, pp. 1868-1872, 2010.

[19] A. Ghorbani and S. Momani, "An effective variational iteration algorithm for solving Riccati differential equations," Applied Mathematics Letters, vol. 23, no. 8, pp. 922-927, 2010.

[20] M. Tatari and M. Dehghan, "Improvement of He's variational iteration method for solving systems of differential equations," Computers \& Mathematics with Applications, vol. 58, no. 11-12, pp. 2160-2166, 2009.

[21] E. Hesameddini and H. Latifizadeh, "Reconstruction of variational iteration algorithms using the Laplace transform," International Journal of Nonlinear Sciences and Numerical Simulation, vol. 10, no. 11-12, pp. 1377-1382, 2009.

[22] G.-C. Wu and D. Baleanu, "Variational iteration method for the Burgers' flow with fractional derivatives-new Lagrange multipliers," Applied Mathematical Modelling, vol. 37, no. 9, pp. 6183-6190, 2013

[23] Y. Khan, N. Faraz, A. Yildirim, and Q. Wu, "Fractional variational iteration method for fractional initial-boundary value problems arising in the application of nonlinear science," Computers \& Mathematics with Applications, vol. 62, no. 5, pp. 2273-2278, 2011.

[24] G.-C. Wu and E. W. M. Lee, "Fractional variational iteration method and its application," Physics Letters A, vol. 374, no. 25, pp. 2506-2509, 2010.

[25] S. Guo and L. Mei, "The fractional variational iteration method using He’s polynomials," Physics Letters A, vol. 375, no. 3, pp. 309-313, 2011.

[26] G.-C. Wu, "A fractional variational iteration method for solving fractional nonlinear differential equations," Computers \& Mathematics with Applications, vol. 61, no. 8, pp. 2186-2190, 2011.

[27] J. Ji, J. Zhang, and Y. Dong, "The fractional variational iteration method improved with the Adomian series," Applied Mathematics Letters, vol. 25, no. 12, pp. 2223-2226, 2012.

[28] G.-C. Wu, "Challenge in the variational iteration method-a new approach to identification of the Lagrange multipliers," Journal of King Saud University, vol. 25, no. 2, pp. 175-178, 2013.

[29] J. H. He, "Asymptotic methods for solitary solutions and compactons," Abstract and Applied Analysis, vol. 2012, Article ID 916793, 130 pages, 2012.

[30] X.-W. Zhou and L. Yao, "The variational iteration method for Cauchy problems," Computers \& Mathematics with Applications, vol. 60, no. 3, pp. 756-760, 2010.

[31] R. C. Mcowen, Partial Differential Equations: Methods and Applications, Prentice Hall, 1996. 


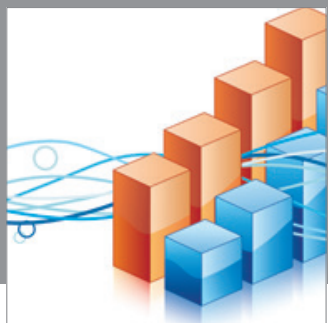

Advances in

Operations Research

mansans

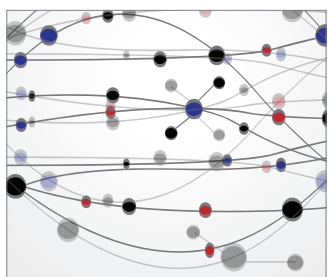

The Scientific World Journal
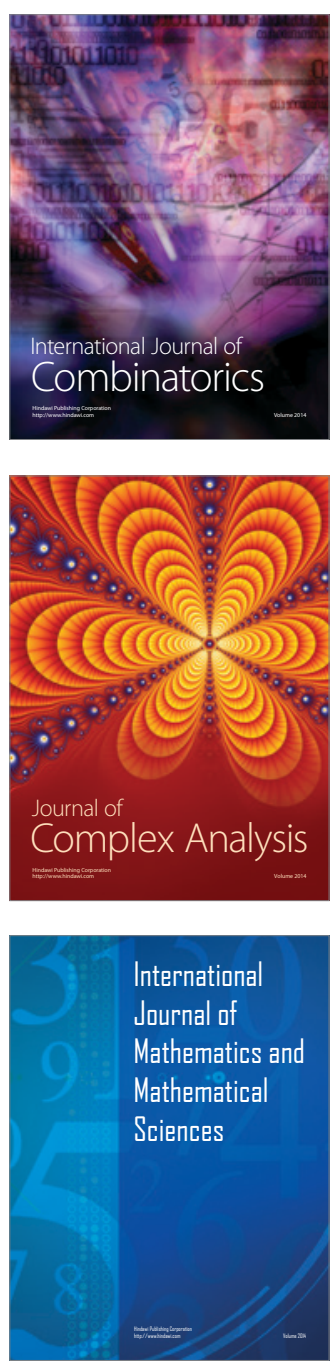
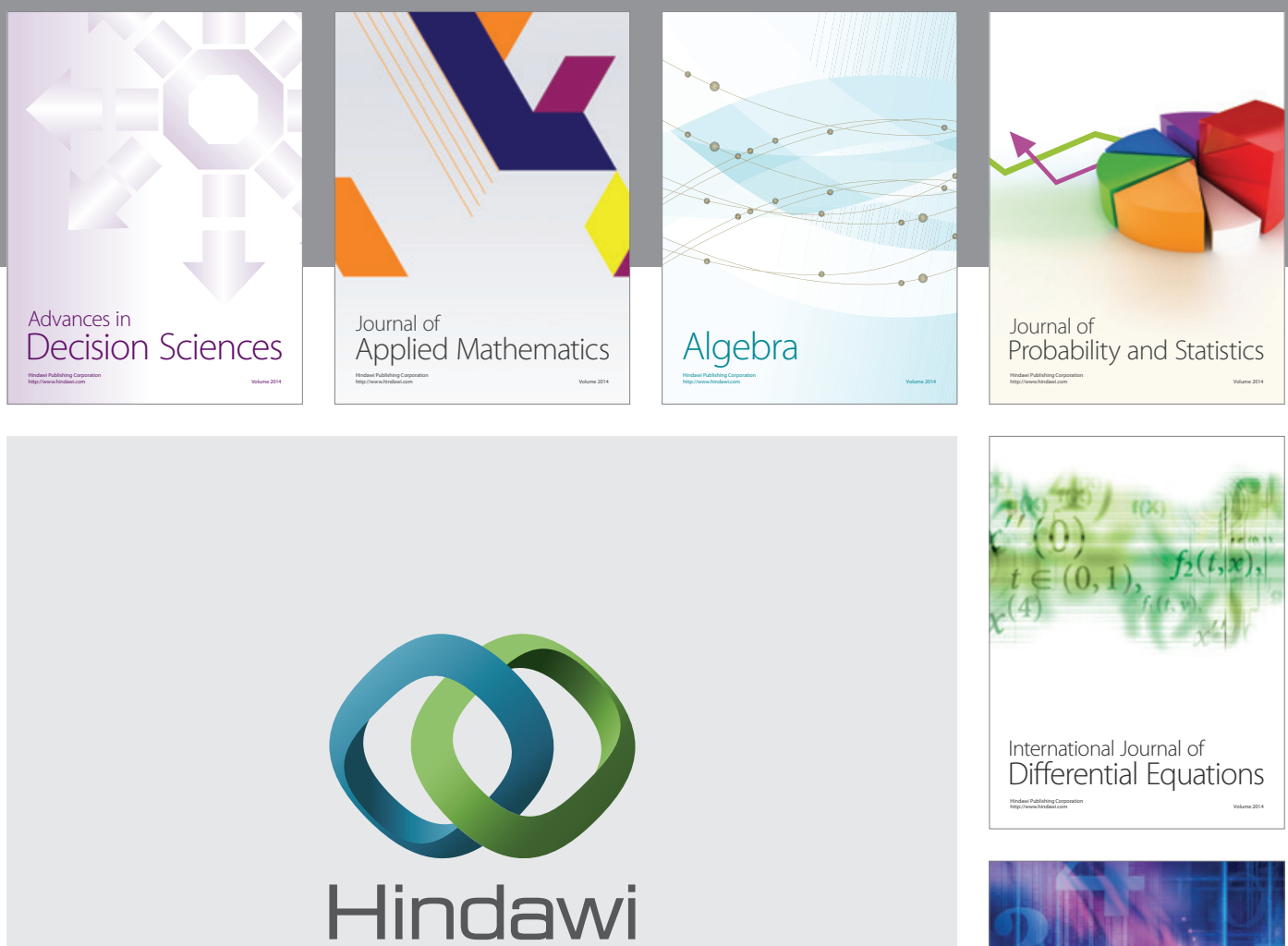

Submit your manuscripts at http://www.hindawi.com
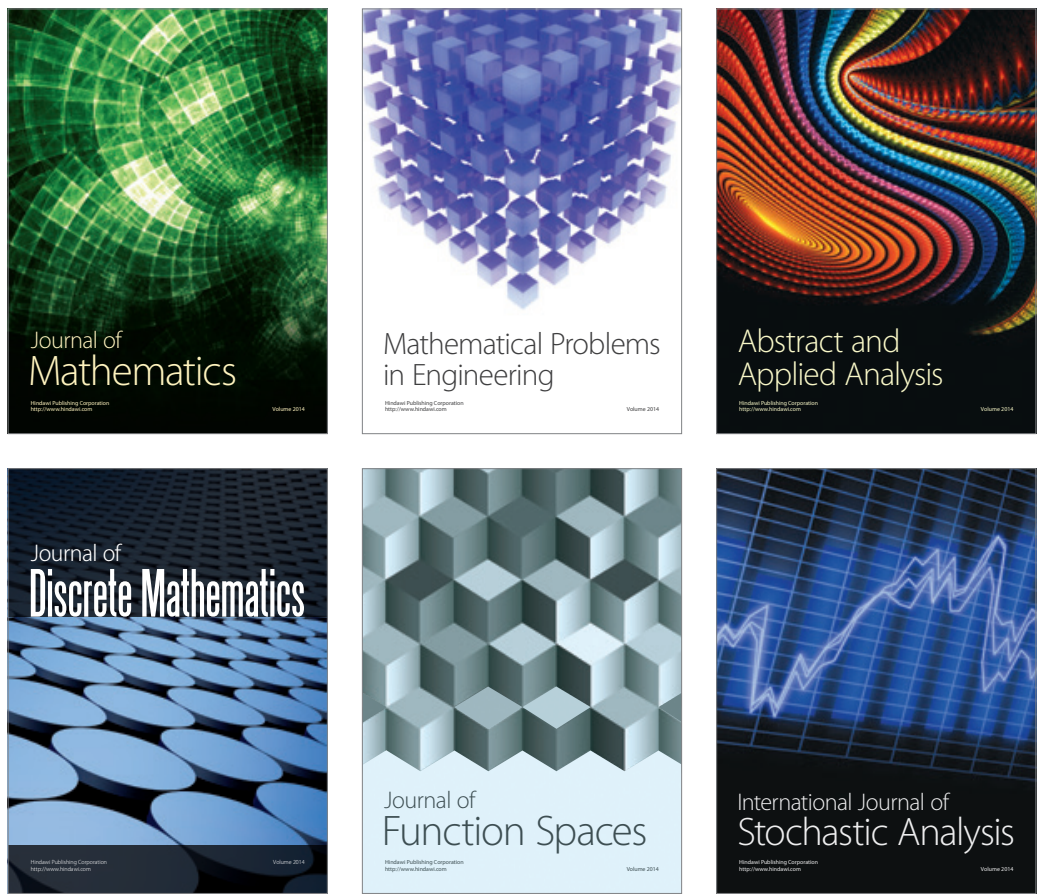

Journal of

Function Spaces

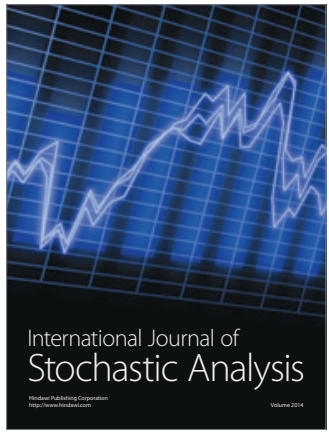

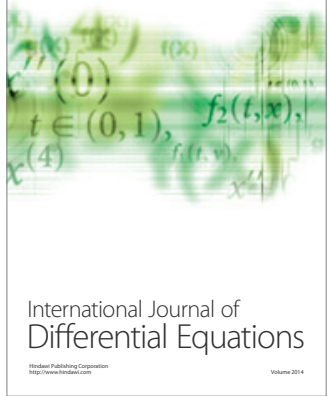
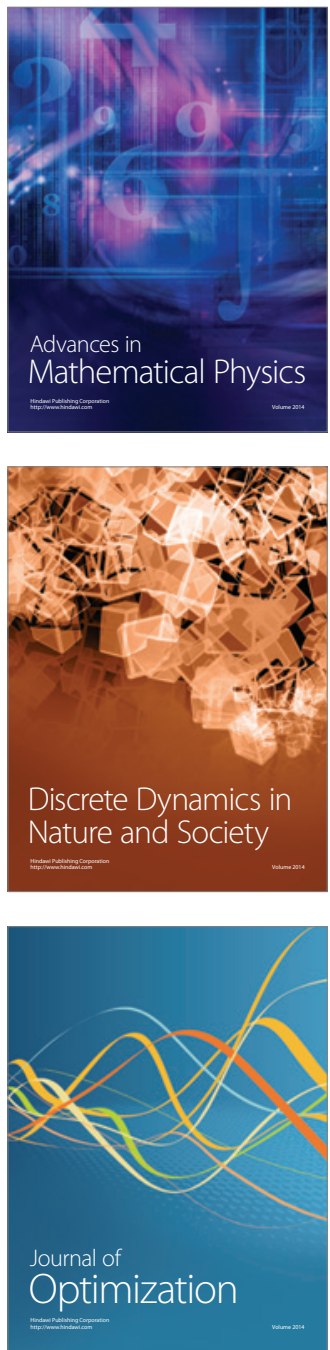